\title{
Radical prostatectomy as radical cure of prostate cancer in a high-risk group: A single-institution experience
}

\author{
NOBUKI FURUBAYASHI ${ }^{1}$, MOTONOBU NAKAMURA ${ }^{1}$, KEN HISHIKAWA ${ }^{1}$, ATSUSHI FUKUDA ${ }^{1}$, \\ TAKASHI MATSUMOTO ${ }^{1}$, KENICHI NISHIYAMA ${ }^{2}$, TAKEHARU YAMANAKA ${ }^{3}$ and YOSHIHIRO HASEGAWA ${ }^{1}$
}

\author{
Departments of ${ }^{1}$ Urology and ${ }^{2}$ Pathology and ${ }^{3}$ Institute for Clinical Research, \\ National Kyushu Cancer Center, Fukuoka, Japan
}

Received May 28, 2012; Accepted September 26, 2012

DOI: $10.3892 / \mathrm{mco} .2012 .39$

\begin{abstract}
This study aimed to evaluate the possibility of performing radical prostatectomy (RP) alone to achieve radical cure of prostate cancer in a high-risk group. Between August 1998 and December 2008, 436 Japanese patients underwent antegrade RP following the exclusion of 139 patients. According to the D'Amico criteria, the low-, intermediate- and high-risk groups comprised 63, 122 and 112 patients, respectively. Twenty-five patients who were classified into the high-risk group based only on T2c stage, were evaluated as a separate intermediate/high-risk group. Results of the multivariate analysis revealed that of the preoperative characteristics only a biopsy Gleason score was a significant predictor in patients with and without PSA failure $(\mathrm{P}=0.017)$. After a median follow-up period of 60 months, the PSA failure-free rates in the low-, intermediate-, high- and intermediate/high-risk groups were $96.5,92.2,76.8$ and $95.0 \%$, respectively. No statistically significant difference was detected in the high- and intermediate/high-risk groups $(\mathrm{P}=0.064)$. Thus, patients classified into the high-risk group based on CT2 stage only, are considered to be potentially eligible for radical treatment by surgery alone, and should not be evaluated as high-risk patients.
\end{abstract}

\section{Introduction}

In prostate cancer, as in other malignancies, it is important to assess the degree of malignancy or the prognosis of patients in order to determine the appropriate treatment. Risk classification, the grouping of patients on the basis of combining several clinical factors, is being widely used in the clinical setting. Several pre-treatment risk classification models for prostate cancer have been proposed thus far, with the D'Amico classification being the most widely-used one. According to this

Correspondence to: Dr Nobuki Furubayashi, Department of Urology, National Kyushu Cancer Center, Notame 3-1-1, Minami-ku, Fukuoka 811-1395, Japan

E-mail: furubayashi.n@nk-cc.go.jp

Key words: prostate cancer, radical prostatectomy, D'Amico classification, high-risk group, prostate-specific antigen failure classification model, in high-risk prostate cancer patients the probability of recurrence subsequent to local treatment alone is low (1). However, the present study aimed to assess the outcome of radical prostatectomy (RP) in high-risk patients with no pre-surgical treatment, with a view to investigate the possibility of complete cure by RP alone in Japanese high-risk prostate cancer patients.

\section{Materials and methods}

Patient characteristics and risk-group classification. The patients underwent prostate biopsy and were diagnosed with prostate cancer in the National Kyushu Cancer Center (Fukuoka, Japan) and at additional associated institutions. Tissue specimens, obtained from 436 patients between August 1998 and December 2008 were reviewed in embedded whole-mount antegrade RP specimens with adenocarcinoma. The patients underwent pelvic lymph node dissection during the same time period. One hundred and thirty-nine patients were excluded from this study, 69 patients due to a past hormonal therapy, 6 patients due to an unclear biopsy or prostatectomy specimen, and 64 patients due to an orchiectomy during RP. At our institution, the one patient classified into the high-risk group according to the D'Amico criteria underwent an orchiectomy during the same time period until December 2004. The patients were Japanese, (median age, 67 years; range, 47-77) and the value of the prostate-specific antigen (PSA) ranged from 0.8 to $88.0 \mathrm{ng} / \mathrm{ml}$ (median, $7.4 \mathrm{ng} / \mathrm{ml}$ ). A median follow-up period after surgery was 60 months.

The patients were classified into three risk groups according to the D'Amico criteria. The low- (PSA $<10$ and Gleason score $\leq 6$ and T1-T2a), intermediate- (PSA, 10.1-20.0 and/or Gleason score, 7 and/or T2b) and high-risk (PSA $>20$ or Gleason score $\geq 8$ or T2c) groups comprised 63 (21.2\%), 122 (41.1\%) and $112(37.7 \%)$ patients, respectively. Additional analyses were carried out using the more restrictive definition according to which clinical stage T2c is an intermediate- rather than a high-risk group characteristic. Twenty-five patients likely to be classified into the high-risk group by the standard definition and into the intermediate-risk group by the more restrictive definition were evaluated as a separate 'intermediate/high' group. One pathologist evaluated the degree of malignancy of the biopsy and prostatectomy specimens according to the 
Table I. Clinicopathological characteristics according to risk group classification.

\begin{tabular}{|c|c|c|c|}
\hline \multirow[b]{2}{*}{ Characteristics } & \multicolumn{3}{|c|}{ Risk groups } \\
\hline & Low & Intermediate & High \\
\hline Total no. of patients & 63 & 122 & 112 \\
\hline Median age, years (range) & $66(47-77)$ & $67(52-76)$ & $67(48-77)$ \\
\hline \multicolumn{4}{|l|}{ Clinical stage, n (\%) } \\
\hline $\mathrm{T} 1 \mathrm{ab}$ & 0 & $3(2.4)$ & $3(2.6)$ \\
\hline $\mathrm{T} 1 \mathrm{c}$ & $42(66.7)$ & $64(52.5)$ & $41(36.6)$ \\
\hline $\mathrm{T} 2 \mathrm{ab}$ & $21(33.3)$ & $55(45.1)$ & $30(26.8)$ \\
\hline $\mathrm{T} 2 \mathrm{c}$ & - & 0 & $33(29.5)$ \\
\hline $\mathrm{T} 3$ & - & - & $5(4.5)$ \\
\hline \multicolumn{4}{|l|}{ Preoperative PSA, n (\%) } \\
\hline$\leq 4.0$ & $8(12.7)$ & $12(9.9)$ & $6(5.4)$ \\
\hline $4.1-10.0$ & $55(87.3)$ & $78(63.9)$ & $64(57.1)$ \\
\hline $10.1-20.0$ & - & $32(26.2)$ & $29(25.9)$ \\
\hline$>20.1$ & - & - & $13(11.6)$ \\
\hline \multicolumn{4}{|l|}{ Biopsy Gleason score, n (\%) } \\
\hline 5 & $8(12.7)$ & - & $2(1.8)$ \\
\hline 6 & $55(87.3)$ & $12(9.8)$ & $5(4.5)$ \\
\hline 7 & - & $110(90.2)$ & $26(23.2)$ \\
\hline 8 & - & - & $27(24.1)$ \\
\hline 9 & - & - & $49(43.7)$ \\
\hline 10 & - & - & $3(2.7)$ \\
\hline \multicolumn{4}{|l|}{ Final Gleason score, n (\%) } \\
\hline 5 & $2(3.2)$ & $1(0.8)$ & $2(1.8)$ \\
\hline 6 & $15(23.8)$ & $11(9.0)$ & $5(4.5)$ \\
\hline 7 & $37(58.7)$ & $91(74.6)$ & $55(49.1)$ \\
\hline 8 & $6(9.5)$ & $6(4.9)$ & $12(10.7)$ \\
\hline 9 & $3(4.8)$ & $13(10.7)$ & $38(33.9)$ \\
\hline \multicolumn{4}{|l|}{ Pathological stage, n (\%) } \\
\hline $\mathrm{pT} 2 \mathrm{ab}$ & $9(14.3)$ & $18(14.8)$ & $7(6.3)$ \\
\hline $\mathrm{pT} 2 \mathrm{c}$ & $47(74.6)$ & $72(59.0)$ & $67(59.8)$ \\
\hline pT3a & $6(9.5)$ & $31(25.4)$ & 29 (25.9) \\
\hline pT3b & $1(1.6)$ & $1(0.8)$ & $9(8.0)$ \\
\hline $\mathrm{pN} 1$ & $1(1.6)$ & 0 & $2(1.8)$ \\
\hline RM1 & $6(9.5)$ & $27(22.1)$ & $22(19.6)$ \\
\hline
\end{tabular}

PSA, prostate-specific antigen; RM, resection margin.

2005 International Society of Urological Pathology (ISUP) Consensus Conference on Gleason grading system (2) and pathological stage based on the 2009 TNM classification (3).

Methods. Prostatectomy specimens were stained and fixed in $10 \%$ neutral-buffered formalin (NBF). The prostate was sectioned into $3-\mathrm{mm}$ sections in the plane perpendicular to the long axis of the gland, from the prostate apex to the tip of the seminal vesicles, followed by hematoxylin and eosin (H\&E) staining and determination of extra prostatic extension (EPE). EPE was defined as a tumor extending from the prostate to the periprostatic soft tissue. The presence of tumor cells at the stained margin of the resection was evaluated as a positive resection margin (pRM). Organ-confined disease (OCD) was defined as pT2 without lymph node metastasis, and specimen-confined disease (SCD) was defined as either pT2 or pT3 without pRM or lymph node metastasis. The follow-up schedule following RP involved a PSA assay every 3 months for the first 2 years, every 4 months for the next 3 years and every 6 month thereafter. Disease recurrence or PSA failure was determined as the time point when the serum PSA level was $>0.2 \mathrm{ng} / \mathrm{ml}$, or RP was performed if the PSA did not decrease below $0.2 \mathrm{ng} / \mathrm{ml}$ after surgery. A number of patients that underwent RP were subsequently treated with radiation and/or hormone therapy prior to the serum PSA level exceeding $0.2 \mathrm{ng} / \mathrm{ml}$. Therefore, for these patients the 
Table II. Correlation between characteristics and PSA failure in the high-risk group.

\begin{tabular}{lccc}
\hline Characteristics & Hazard ratio & P-value & $95 \% \mathrm{Cl}$ \\
\hline Univariate analysis & & & 0.824 \\
Age $<70$ vs. $\geq 70$ years & 0.903 & 0.362 & $0.368-2.217$ \\
PSA & 1.012 & 0.065 & $0.986-1.039$ \\
Biopsy Gleason score $\leq 7$ vs. $\geq 8$ & 3.953 & 0.325 & $0.917-17.040$ \\
cT1c vs. cT2 or cT3 & 0.618 & $0.028^{\mathrm{a}}$ & $0.237-1.612$ \\
RP Gleason score $\leq 7$ vs. $\geq 8$ & 2.917 & $0.007^{\mathrm{a}}$ & $1.119-7.600$ \\
Organ-confined vs. non-organ-confined & 3.379 & $<0.001^{\mathrm{a}}$ & $1.378-8.286$ \\
Specimen-confined vs. non-specimen-confined & 4.718 & & $1.955-11.389$ \\
Multivariate analysis & & $0.017^{\mathrm{a}}$ & \\
Biopsy Gleason score $\leq 7$ vs. $\geq 8$ & 4.332 & $<0.001^{\mathrm{a}}$ & $1.247-27.289$ \\
Specimen-confined vs. non-specimen-confined & 5.024 & & $2.047-12.337$ \\
\hline
\end{tabular}

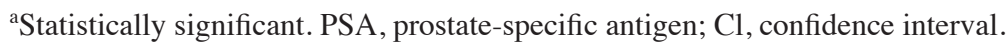

time point of the adjuvant therapy was defined as the date of disease recurrence.

Statistical analysis. Statistical analyses were carried out using the $\mathrm{JMP}^{\circledR}$ version 8 software (SAS Institute, Inc., Cary, NC, USA). The PSA failure-free rate was determined using the Kaplan-Meier method. The significance of the clinicopathological parameters associated with PSA failure was assessed using the Cox proportional hazards regression model. The log-rank test was used to determine differences among each risk group. $\mathrm{P}<0.05$ was considered to indicate a statistically significant difference.

\section{Results}

Clinicopathological characteristics according to risk group classificaction. The clinicopathological characteristics of the three risk groups are shown in Table I. According to the D'Amico criteria, the low- (PSA $<10$ and Gleason score $\leq 6$ and T1-T2a), intermediate- (PSA, 10.1-20.0 and/or Gleason score, 7 and/or T2b) and high-risk (PSA $>20$ or Gleason score $\geq 8$ or T2c) groups comprised $63(21.2 \%), 122(41.1 \%)$ and 112 $(37.7 \%)$ patients, respectively. No differences were observed in the age of the patients in the groups. According to the RP Gleason score, the low-, intermediate- and high-risk groups had high-grade (Gleason score $\geq 8$ ) tumors in 14.3 (9/63), 15.6 $(19 / 122)$ and $44.6 \%(50 / 112)$ of patients, respectively. The tumor was organ-confined in 88.9 (56/63), 73.8 (90/122) and $65.2 \%(73 / 112)$ of patients, respectively. Only one patient with lymph node metastasis in the high-risk group was staged pT2. Lymph node involvement was observed in one patient in the low-risk group and in two patients in the high-risk group.

Concerning the high-risk group, the median PSA prior to surgery was $8.6 \mathrm{ng} / \mathrm{ml}$. Seventy-nine patients $(70.5 \%)$ had a biopsy Gleason score of $\geq 8$. Forty-four patients $(39.2 \%)$ had non-palpable disease (cT1c). The Gleason scores for the RP specimens were lower than those for the biopsy scores (downgraded) in 46 patients $(41.1 \%)$ and higher (upgraded) than those for the biopsy scores in 20 patients (17.9\%). Seven

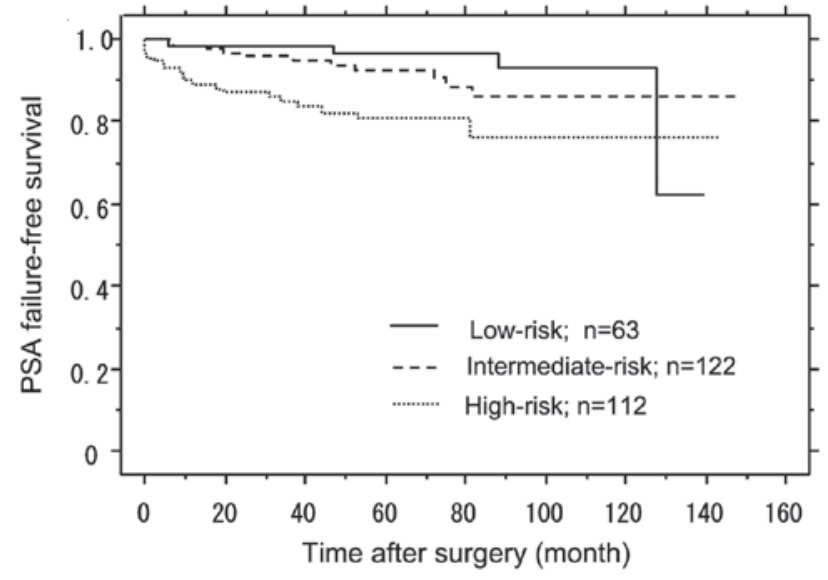

Figure 1. Kaplan-Meier estimates of PSA failure-free survival, according to the risk group $(\mathrm{P}=0.005 ; \mathrm{df}=2)$ are shown. PSA, prostate-specific antigen.

patients $(15.2 \%)$ of the downgraded groups had PSA failure, whereas 3 patients $(15.0 \%)$ of the upgraded and $10(21.7 \%)$ of the same-graded groups had PSA failure. Two patients from the same-graded groups with PSA failure exhibited lymph node metastasis.

Adjuvant therapy subsequent to RP. Thirty-five patients had PSA failure subsequent to RP. The PSA level in 4 patients did not decrease below $0.2 \mathrm{ng} / \mathrm{ml}$ after surgery and, therefore, 4 of these patients received adjuvant therapy. The PSA level of 21 patients was $>0.2 \mathrm{ng} / \mathrm{ml}$ after surgery, while 14 patients received adjuvant therapy. Ten patients received adjuvant therapy prior to the serum PSA level reaching $>0.2 \mathrm{ng} / \mathrm{ml}$.

PSA failure-free survival according to risk group classification. After the median follow-up period of 60 months, the PSA failure-free rate in the low-, intermediate- and high-risk groups was 96.5, 92.2 and 80.6\%, respectively (Fig. 1). The difference between the high- and intermediate-risk groups was statistically significant, according to the log-rank test $(\mathrm{P}=0.017)$ (Fig. 1). The difference between the high- and 


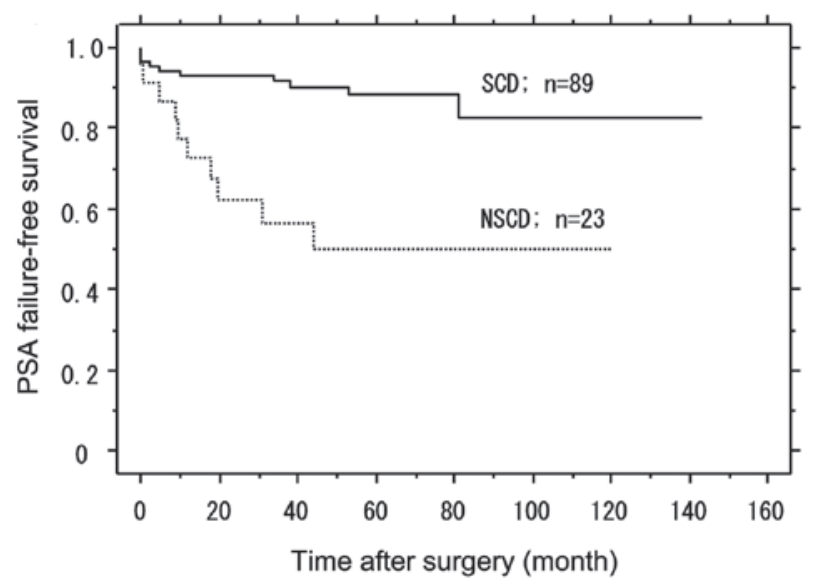

Figure 2. Kaplan-Meier estimates of PSA failure-free survival, according to $\mathrm{SCD}$ in the high-risk group $(\mathrm{P}<0.001 ; \mathrm{df}=1)$ are shown. $\mathrm{PSA}$, prostate-specific antigen; SCD, specimen-confined disease; NSDC, non-specimen-confined disease.

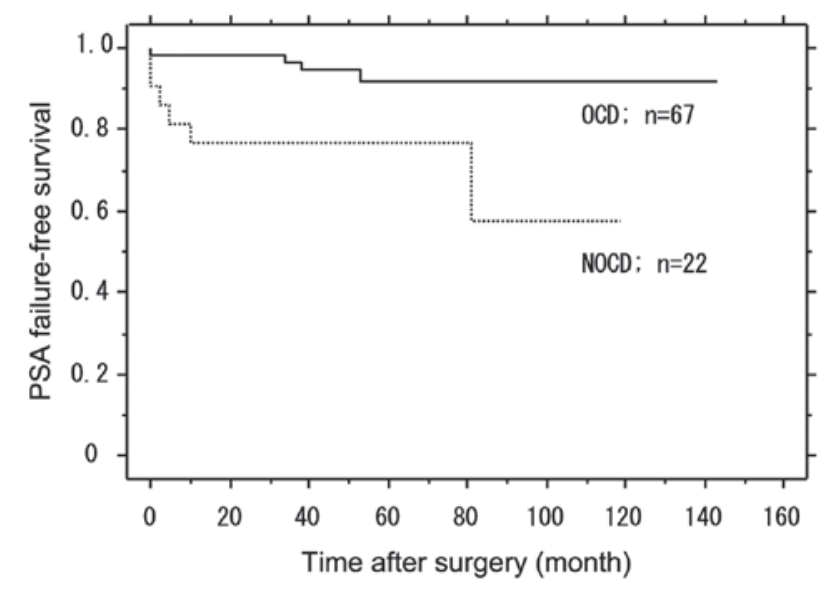

Figure 3. Kaplan-Meier estimates of PSA failure-free survival, according to OCD among SCD in the high-risk group $(\mathrm{P}=0.004 ; \mathrm{df}=1)$ are shown. PSA, prostate-specific antigen; OCD, organ-confined disease; NOCD, non-organ-confined disease.

low-risk groups was statistically significant, according to the log-rank test $(\mathrm{P}=0.009)$ (Fig 1).

Correlation between the characteristics and PSA failure in the high-risk group. The correlation between the characteristics and PSA failure in the high-risk group is shown in Table II. According to the Cox proportional hazards analysis of the high-risk group, pre-operative variables, such as age, pre-operative PSA, biopsy Gleason score and clinical tumor stage were not significant predictors, and only post-operative characteristics, such as the RP Gleason score, organ-confined and specimen-confined status were significant predictors based on the univariate analysis. In the multivariate analysis, statistically significant differences were found in the biopsy Gleason score and specimen-confined status in the patients with and without PSA failure.

PSA failure-free survival according to SCD in the high-risk group. SCD was determined as either pT2 to pT3 without pRM or lymph node metastasis, and it comprised $79.5 \%(89 / 112)$

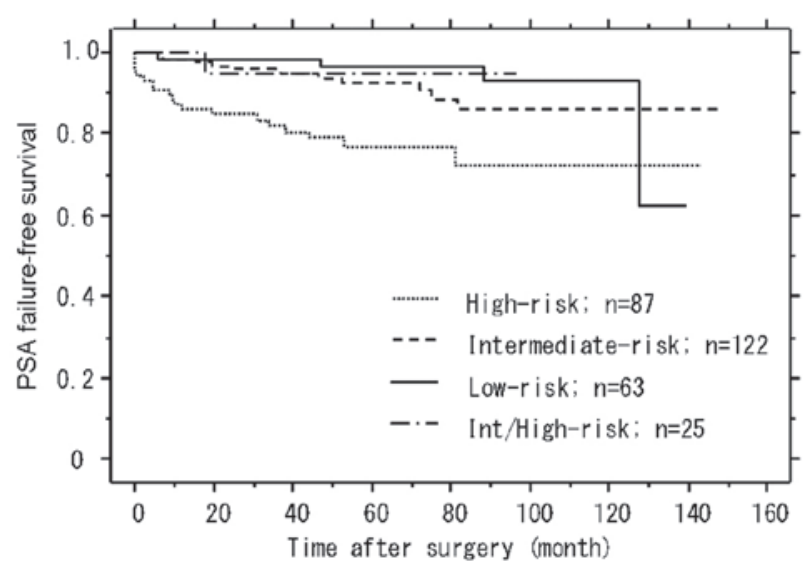

Figure 4. Kaplan-Meier estimates of PSA failure-free survival, according to risk group under the restrictive definition $(\mathrm{P}<0.001 ; \mathrm{df}=3)$ are shown. PSA, prostate-specific antigen.

of patients in the high-risk group. The PSA failure-free survival with SCD was significantly higher than in those with non-specimen-confined disease (NSCD) $(\mathrm{P}<0.001)$. Patients $(49.8 \%)$ with NSCD had PSA failure, while in patients with SCD the PSA failure-free rate was $88.2 \%$ following a median follow-up of 60 months (Fig. 2).

PSA failure-free survival according to the OCD among SCD in the high-risk group. OCD was defined as pT2 without lymph node metastasis. Among the SCD in the high-risk group, OCD was identified in $75.3 \%(67 / 89)$ of the patients. The PSA failure-free survival with OCD was significantly higher compared to patients with non-OCD $(\mathrm{P}=0.004)$. Patients with non-OCD $(23.4 \%)$ had PSA failure, while in those with OCD the PSA failure-free rate was $92.1 \%$ following a median follow-up of 60 months (Fig. 3).

A more restrictive definition assigns clinical stage T2c to intermediate-rather than high-risk patients. Patients likely to be classified as high-risk patients by the standard definition and intermediate-risk patients by the more restrictive definition were evaluated as a separate 'intermediate/high' group. The intermediate/high-risk group comprised 25 patients, belonging to the high-risk group by the standard definition. By the restrictive definition, the low-, intermediate-, high- and intermediate/high-risk groups comprised 63 (21.2\%), 122 (41.1\%), 87 (29.3\%) and $25(8.4 \%)$ patients, respectively. In the intermediate/high-risk group, only one patient exhibited PSA failure following surgery. After a median follow-up period of 60 months, the PSA failure-free rates in the low-, intermediate-, high- and intermediate/high-risk groups were 96.5, 92.2, 76.8 and $95.0 \%$, respectively. The difference between the high- and intermediate/high-risk groups was not statistically significant by the log-rank test $(\mathrm{P}=0.064)$ (Fig. 4).

\section{Discussion}

High-risk prostate cancer classified according to the D'Amico criteria is an important disease, accounting for 20-35\% of localized prostate cancer cases $(4,5)$. However, generally speaking, RP alone cannot achieve satisfactory PSA control 
(1). Nevertheless, individual disease state characteristics of the high-risk group are not necessarily uniform and RP is also known to be likely to have good cure rates. The present study aimed to retrospectively assess the outcome of RP in high-risk patients with no pre-surgical treatment, with a view to investigate the possibility of complete cure by RP alone in Japanese high-risk prostate cancer patients

As shown in Table I, the patients were classified into three groups according to the D'Amico criteria: low-, intermediateand high-risk groups, accounting for $21.2 \%$ (63/297), $41.4 \%$ $(122 / 297)$ and $37.7 \%(112 / 297)$, respectively. In addition, while those cases in the high-risk group with biopsy specimen Gleason scores of $\geq 8$ accounted for $70.5 \%$ (79/112) of this group, the proportion of cases with a Gleason score from RP specimens of $\geq 8$ in the low-, intermediate- and high-risk groups was $14.3 \%$ (9/63), 15.6\% (19/122) and 44.6\% (50/112), respectively. Recent studies have demonstrated that $>1 / 3$ of the patients with a Gleason score of $8-10$, according to the biopsy findings are likely to have a Gleason score of $\leq 7$ in the RP specimen $(6,7)$. In the high-risk group of the present study, $48.1 \%(38 / 79)$ with a Gleason score of $8-10$ on biopsy had a Gleason score of $\leq 7$ in the RP specimen. These findings suggest that a number of the cases classified into the high-risk group according to the D'Amico criteria in reference to the preoperative factor, i.e., Gleason score on biopsy, are considered to have been downgraded histopathologically with respect to their excised specimens and are the cases in which $\mathrm{RP}$ alone may result in good cure rates. However, the difference between patients with a Gleason score of $\leq 7$ in the RP specimen and patients with a Gleason score of $\geq 8$ in the RP specimen was not statistically significant with regard to the PSA failure-free survival, based on results of the log-rank test $(\mathrm{P}=0.080)$, among the patients with a Gleason score of 8-10 on biopsy of high-risk group. Consequently, the cases with an excised specimen Gleason score downgraded with regard to the preoperative factor were not considered to be cases in which complete cure may be achieved via surgery alone.

PSA failure-free survival rates were examined for each group and yielded the following results: $93.7 \%$ (59/63) for the low-risk group; $91.0 \%$ (111/122) for the intermediate-risk group and $82.1 \%$ (92/112) for the high-risk group (Fig. 1). In their study, Kawamorita et al (8) investigated these rates only in Japanese patients and concluded that the PSA failure-free rates in the low-, intermediate- and high-risk groups were $87.8,87.3$ and $64.5 \%$, respectively. Compared to these findings, although the PSA failure-free survival rates at this institution following RP alone for the high-risk group were good, compared to the low- and intermediate-risk groups, the PSA failure-free survival rates for the high-risk group were low $(\mathrm{P}=0.017,0.009)$. The reason for this difference was that the results included cases in which control via surgery alone is difficult. Nevertheless, urologists are aware of the fact that in several high-risk cases, the treatment outcomes of surgery alone are good. By contrast, the correlation between the characteristics and PSA failure were examined in the high-risk group (Table II). According to results of the multivariate analysis, only the biopsy Gleason score was found to be a significant predictor in patients with and without PSA failure $(\mathrm{P}=0.017)$, among the pre-operative variables. Results of the univariate and multivariate analyses did not reveal statisti- cally significant differences in preoperative variables, such as pre-operative PSA and clinical tumor stage that were risk profiles in the D'Amico risk classification $(\mathrm{P}=0.362, \mathrm{P}=0.325)$. Post-operative variables, such as the RP Gleason score and the organ-confined status, were found to be significant predictors based on the univariate analysis $(\mathrm{P}=0.028, \mathrm{P}=0.007)$, while a post-operative variable, such as specimen-confined status was alone a significant predictor, in the univariate and multivariate analyses $(\mathrm{P}<0.001)$.

Fig. 2 shows PSA failure-free survival according to SCD, indicating that the PSA failure-free rate was $88.8 \%(79 / 89)$ in patients with NSCD, while PSA failure-free survival with SCD was significantly higher compared to patients with NSCD $(\mathrm{P}<0.001)$. Mian et al $(9)$ reported that among 188 patients with high-grade cancer, the subgroup with SCD had an $84 \%$ PSA failure-free rate and emphasized that it is important to resect high-risk prostate cancer completely by RP. In this study, among the patients with SCD, those with OCD had a higher PSA failure-free rate compared to the patients with non-organ-confined disease (NOCD). In this study, of the 112 patients in the high-risk group, the subgroup with SCD had an $88.2 \%$ PSA failure-free rate, following a median follow-up of 60 months (Fig. 2). Of the patients with SCD, those with OCD had a higher PSA failure-free rate compared to patients with NOCD ( $\mathrm{P}=0.004)$ (Fig. 3). Therefore, the selection of patients who are expected to have pathologically OCD is especially important for the surgical treatment of high-risk disease.

Byar et al (10) reported that when pathologically examined, tumors apparently unilateral on rectal examination are bilateral in $\sim 70 \%$ of patients, whereas adenocarcinoma of the prostate is multifocal in $>85 \%$ of patients. Additionally, we often experience cases in which, although a prostate biopsy detects cancer in a unilateral lobe of the prostate, testing of the excised specimens demonstrates a prostate cancer in the bilateral lobes. In the present study, 97 patients were diagnosed with prostate cancer in the bilateral prostate lobe in the prostatectomy specimen out of the 106 patients considered to have unilateral cancer based on the findings of rectal examinations. Prostate biopsies were performed in 1,580 cases during the same period as this study. RP was performed in 154/287 cases in which cancer was detected only in a unilateral lobe of the prostate, and the surgical findings demonstrated prostate cancer in the bilateral lobes in 128 cases $(83.1 \%)$. Thus, these findings do not confirm whether or not cT2c should be used to identify high-risk patients.

Of those cases classified into the high-risk group according to the D'Amico criteria, we re-classified the high-risk ones selected due exclusively to their cT2c stage, in order to re-examine the PSA failure-free survival (Fig. 4). Using the more restrictive, definition-assigned clinical stage T2c patients as intermediate/high-risk within the high-risk group, 25 patients $(8.4 \%)$ were assigned to the intermediate/high-risk group and 87 patients (29.3\%) to the high-risk group. After a median follow-up period of 60 months, the PSA failure-free rates in the low-, intermediate-, high- and intermediate/ high-risk groups were 96.5, 92.2, 76.8 and $95.0 \%$, respectively. However, the difference between the high- and intermediate/ high-risk groups was not statistically significant, based on the log-rank test $(\mathrm{P}=0.064)$. This is the reason for the small number 
of patients in the intermediate/high-risk group compared to the high-risk group. These findings demonstrate, that the outcomes of the cases classified into the standard high-risk group due exclusively to their cT2c stage, are good even with $\mathrm{RP}$ alone. It is, therefore, believed that by excluding the cases classified into the standard high-risk group due exclusively to their cT2c stage, the cases in which a complete cure is difficult to achieve via RP alone should be considered to belong to the high-risk group. In their study, Cooperberg et al (11) reported that the clinical stage T2c alone should not classify a patient into the high-risk group. Patients likely to be classified into the high-risk group based only on the presence of T2c stage disease had a markedly lower risk of recurrence compared to patients evaluated as high-risk patients using the more restrictive definition, as well as to patients classified into the intermediate-risk group. These descriptions are consistent with the observations in the present study. Although RP is not recommended for the high-risk patients, the patients classified into the high-risk group on the basis of their T2c stage only are likely to benefit more from treatment by RP alone.

We retrospectively assessed the outcome of RP alone in Japanese patients with high-risk prostate cancer. The cases classified into the high-risk group based on cT2c stage only are believed to be patients likely to achieve complete cure via surgery alone. Consequently, such cases should not be evaluated as high-risk cases, based on their cT2c stage only, according to the risk classification system.

\section{References}

1. D'Amico AV, Whittington R, Malkowicz SB, et al: Biochemical outcome after radical prostatectomy, external beam radiation therapy, or interstitial radiation therapy for clinically localized prostate cancer. JAMA 280: 969-974, 1998.
2. Epstein JI, Allsbrook WC Jr, Amin MB and Eqevad LL; ISUP Grading Committee: The 2005 International Society of Urological Pathology (ISUP) Consensus Conference on Gleason Grading of prostatic carcinoma. Am J Surg Pathol 29: 1228-1242, 2005.

3. Sobin LH, Gospodarowicz MK and Wittekind Ch (eds): TNM Classification of Malignant Tumors. 7th edition. Wiley-Blackwell, Oxford, 2009.

4. Grossfeld GD, Latini DM, Lubeck DP, Broering JM, Li YP, Mehta SS and Carroll PR: Predicting disease recurrence in intermediate and high-risk patients undergoing radical prostatectomy using percent positive biopsies: results from CaPSURE. Urology 59: 560-565, 2002

5. D'Amico AV, Whittington R, Malkowicz SB, et al: Predicting prostate specific antigen outcome preoperatively in the prostate specific antigen era. J Urol 166: 2185-2188, 2001.

6. Manoharan M, Bird VG, Kim SS, Civantos F and Soloway MS: Outcome after radical prostatectomy with a pretreatment prostate biopsy Gleason score of $>/=8$. BJU Int 92: 539-544, 2003.

7. Boorjian SA, Kames RJ, Crispen PL, Ragel LJ, Berqstralh EJ, Sebo TJ and Blute ML: The impact of discordance between biopsy and pathological Gleason scores on survival after radical prostatectomy. J Urol 181: 95-104, 2009.

8. Kawamorita N, Saito S, Ishidoya S, Ito A, Saito H, Kato M and Arai Y: Radical prostatectomy for high-risk prostate cancer: biochemical outcome. Int J Urol 16: 733-738, 2009.

9. Mian BM, Troncoso P, Okihara K, Bhadkamkar V, Johnston D, Reves AO and Babaian RJ: Outcome of patients with Gleason score 8 or higher prostate cancer following radical prostatectomy alone. J Urol 167: 1675-1680, 2002.

10. Byar DP and Mostofi FK: Carcinoma of the prostate: prognostic evaluation of certain pathologic features in 208 radical prostatectomies. Examined by the step-section technique. Cancer 30: $5-13,1972$.

11. Cooperberg MR, Cowan J, Broering JM and Carroll PR: High-risk prostate cancer in the United States, 1990-2007. World J Urol 26: 211-218, 2008. 